\title{
DEEP NEURAL NETWORK MODELING OF UNKNOWN PARTIAL DIFFERENTIAL EQUATIONS IN NODAL SPACE
}

\author{
ZHEN CHEN*, VICTOR CHURCHILL*, KAILIANG WU ${ }^{\dagger}$, AND DONGBIN XIU*
}

\begin{abstract}
We present a numerical framework for deep neural network (DNN) modeling of unknown time-dependent partial differential equation (PDE) using their trajectory data. Unlike the recent work of [Wu and Xiu, J. Comput. Phys. 2020], where the learning takes place in modal/Fourier space, the current method conducts the learning and modeling in physical space and uses measurement data as nodal values. We present a DNN structure that has a direct correspondence to the evolution operator of the underlying PDE, thus establishing the existence of the DNN model. The DNN model also does not require any geometric information of the data nodes. Consequently, a trained DNN defines a predictive model for the underlying unknown PDE over structureless grids. A set of examples, including linear and nonlinear scalar PDE, system of PDEs, in both one dimension and two dimensions, over structured and unstructured grids, are presented to demonstrate the effectiveness of the proposed DNN modeling. Extension to other equations such as differential-integral equation, is also discussed.
\end{abstract}

Key words. Deep neural network, meshfree, governing equation discovery, nodal space

1. Introduction. Data driven modeling and learning of unknown physical laws has attracted much attention in recent years. Most efforts were devoted to learning dynamical systems, i.e., systems of ordinary differential equations (ODEs). One popular approach is to construct a mapping from state variables to their time derivatives. The mapping can be constructed as a sparse approximation from a large dictionary set, such that exact equation recovery can be achieved under certain circumstances. See, for example, 11, and its various extensions [1, 4, 18, 19, 21. The approach has also been applied to recovery of partial differential equations (PDEs) ([14, 17]). The mapping can also be constructed via the more traditional approximation methods such as polynomial approximation. This approach does not achieve exact equation recovery but can ensure high accuracy in the more traditional sense. See [23] and its extension to Hamiltonian systems [22. More recently, deep neural networks (DNNs) have been used to construct the mapping. Studies of this direction include modeling of ODEs [13, 9, 15, as well as PDEs [6, 11, 12, 10, 15, 20].

A different approach for learning an unknown system is to construct a mapping between two system states separated by a short time (9]). For ODEs, the mapping is governed by the flow map of the underlying system. Although not directly recovering the underlying equations, the approach can define an accurate predictive model for the unknown system, so long as an accurate approximation for the flow map is constructed. One practical advantage of the flow map based approach is that it eliminates the need for temporal derivative data, which can be difficult to acquire and may be subject to larger errors. The studies on this approach mostly rely on the use of DNNs. In particular, residual network (ResNet), developed in the image analysis community (3), was found to be highly suitable for recovering the flow map (9]). Since its introduction ([9]), the flow map based DNN modeling approach has been extended to modeling of non-autonomous dynamical systems ([8]), parametric

${ }^{*}$ Department of Mathematics, The Ohio State University, Columbus, OH 43210, USA. Emails: chen.7168@osu.edu, churchill.77@osu.edu, xiu.16@osu.edu. Funding: This work was partially supported by AFOSR FA9550-18-1-0102.

$\dagger^{\dagger}$ Department of Mathematics, Southern University of Science and Technology, Shenzhen 518055, P.R.China. Email: wukl@sustech.edu.cn. 
dynamical systems ([7]), partially observed dynamical systems ([2]), as well as PDE (24).

The focus of this paper is on the development of a general numerical framework for approximating/learning unknown time-dependent PDE. Even though the topic has been explored in several recent articles, cf., 6, 11, 12, 10, 5, 20, the existing studies are relatively limited in scope, in the sense that they have mostly focused on learning certain specific types of PDE or identifying some unknown terms in a given PDE. The contribution of this paper is in the development of a general DNN framework for modeling general types of unknown PDE. More specifically, instead of identifying the various terms in the unknown PDEs, as in most of the existing studies, our proposed method seeks to approximate the evolution operator of the underlying unknown PDE. This can be considered an extension of the flow map based learning for ODE (9]) to PDE. The evolution operator completely characterizes the time evolution of the PDE solution, and its accurate approximation allows one to construct an accurate predictive model for the underlying unknown PDE. The proposed method here is closely related to the recent work of [24. The main difference is that the method of 24] conducts the learning in modal space, i.e., generalized Fourier space, and requires a set of pre-defined basis functions and transformation of measurement data into the modal space. Our current work conducts the learning directly in physical space by using measurement of the solution states on a set of grids/nodes. To achieve this, we propose a new DNN structure, consisting of a disassembly block and an assembly layer, that has a direct correspondence to a general time-stepping evolution of the unknown PDE. Subsequently, the mathematical existence of the DNN model for the unknown PDE can be established. On the practical side, the proposed DNN structure allows one to use structure-free grids/nodes without any geometric information. This can be advantageous for many practical problems, where measurement data are collected at rather arbitrary physical locations. A comprehensive set of examples are presented to demonstrate the effectiveness of the proposed DNN learning. The examples include learning of linear and nonlinear scalar PDE, systems of PDEs, in both one spatial dimension and two spatial dimensions, over structured and unstructured grids. The proposed DNN framework can also be used to learn other types of equations, e.g., differential-integral equation.

This paper is organized as follows. After the problem setup and preliminary materials in Section 2, we present the main approach in Section 3 which includes the mathematical justification in Section 3.1 and our DNN structure in Section 3.2. Upon discussing the model training in Section 3.4 we discuss the extensions to system of PDEs in Section 3.5.1 and other differential equations in Section 3.5.2. Finally, we present numerical examples in Section 4 to demonstrate the properties of the proposed approach.

2. Preliminaries. We first introduce the problem setup and notation for our discussion. For notational clarity, we start the discussion on scalar PDE. Extension to systems of PDEs is straightforward and will be left to the latter part of this paper.

2.1. Problem Setup. Let us consider an autonomous time-dependent PDE,

$$
\begin{cases}u_{t}=\mathcal{L}(u), & (x, t) \in \Omega \times \mathbb{R}^{+}, \\ \mathcal{B}(u)=0, & (x, t) \in \partial \Omega \times \mathbb{R}^{+}, \\ u(x, 0)=u_{0}(x), & x \in \bar{\Omega},\end{cases}
$$


where $\Omega \subset \mathbb{R}^{d}, d=1,2,3$, is the physical domain under consideration, and $\mathcal{L}$ and $\mathcal{B}$ stand for the PDE operator and boundary condition operator, respectively. Our basic assumption is that the PDE is unknown.

We assume data of the state variable $u$ are available over a set of nodal points, or grids,

$$
X_{N}=\left\{x_{1}, \ldots, x_{N}\right\} \subset \Omega,
$$

and by using vector notiation, we write

$$
\mathbf{u}(t)=\left(u\left(x_{1}, t\right), \ldots, u\left(x_{N}, t\right)\right)^{T} .
$$

Also, the data are only available at certain discrete time instances, resulting in socalled snapshots of the solution

$$
\mathbf{u}\left(t_{j}^{(k)}\right), \quad j=1, \ldots, \ell^{(k)}, \quad k=1, \ldots, N_{t r a j} .
$$

Here the superscript $k$ denotes the $k$-th "trajectory", which implies all $\ell^{(k)}$ snapshots are evolved from the same (unknown) initial state, and $N_{t r a j}$ denotes the total number of such "trajectories". We then group the solution snapshots into pairs at two consecutive time instances,

$$
\left(\mathbf{u}\left(t_{j}^{(k)}\right), \quad \mathbf{u}\left(t_{j+1}^{(k)}\right)\right), \quad j=1, \ldots, \ell^{(k)}-1, \quad k=1, \ldots, N_{t r a j} .
$$

For autonomous PDE, the time variable can be arbitrarily shifted. After collecting all the data pairs over all the trajectories and re-ordering them using a single index $m$, we obtain

$$
\left(\mathbf{u}_{m}(0), \mathbf{u}_{m}(\Delta t)\right), \quad m=1, \ldots, M,
$$

where $\Delta t>0$ is the time step between each pair, and $M=\ell^{(1)}+\cdots+\ell^{\left(N_{t r a j}\right)}-N_{t r a j}$ the total number of data pairs. This data set (2.2) shall be our training data set, where we assumed $\Delta t$ as a constant time step only for notational convenience. (Variable time steps can be readily modeled as an additional input parameter, as discussed in [7].) Each pair in the data set can be considered as a solution trajectory of two entries, starting with the "initial condition" $\mathbf{u}_{m}(0)$ and ending with the state $\mathbf{u}_{m}(\Delta t)$ a short time $\Delta t>0$ later.

Our goal is to construct an accurate approximation of the evolution/dynamics of the unknown governing equation (2.1) via the snapshot data (2.2). Once the approximation is constructed, it can serve as a predictive model to provide prediction and analysis of the unknown PDE system.

2.2. Deep Learning of ODE Systems. We now briefly review flow-map based deep learning of ordinary differential equations (ODEs) 9 . The idea behind the flowmap based deep method is used as a starting point of our method for PDE learning.

Consider an unknown dynamical system $d \mathbf{x} / d t=\mathbf{f}(\mathbf{x})$, where $\mathbf{x} \in \mathbb{R}^{n}$ and $\mathbf{f}$ is unknown. Let $\boldsymbol{\Phi}: \mathbb{R}^{n} \times \mathbb{R} \rightarrow \mathbb{R}^{n}$ be the flow map of the system. The state variables satisfy $\mathbf{x}\left(s_{1}\right)=\mathbf{\Phi}\left(\mathbf{x}\left(s_{0}\right), s_{1}-s_{0}\right)$, for any $s_{1} \geq s_{0}$. Suppose data of the state variables $\mathbf{x}$ are available as

$$
\mathbf{x}\left(t_{j}^{(k)}\right), \quad j=1, \ldots, \ell^{(k)}, \quad k=1, \ldots, N_{t r a j},
$$


for a total number of $N_{\text {traj }}$ trajectories over time instances $\left\{t_{j}^{(k)}\right\}$ with step size $\Delta_{j}^{(k)}=t_{j+1}^{(k)}-t_{j}^{(k)}$.

For the flow-map based learning method developed in 9, we first re-group the trajectory data as pairs of two adjacent time instances,

$$
\left\{\mathbf{x}_{m}(0), \mathbf{x}_{m}(\Delta)\right\}, \quad m=1, \ldots, M,
$$

where $M$ is the total number of such data pairs, and for notational convenience the time step $\Delta_{j}^{(k)}$ is assumed to be a constant $\Delta$ for all $j$ and $k$. Note that for autonomous system, time $t$ can be arbitrarily shifted and only the relative time difference is relevant.

We then construct a standard fully connected feedforward deep neural network, whose input and output layers both have $n$ neurons. Let $\mathbf{N}: \mathbb{R}^{n} \rightarrow \mathbb{R}^{n}$ be its associated mapping operator and define residual network (ResNet) mapping ([3])

$$
\mathbf{y}^{\text {out }}=\left[\mathbf{I}_{n}+\mathbf{N}\right]\left(\mathbf{y}^{\text {in }}\right),
$$

where $\mathbf{I}_{n}$ is the identity matrix of size $n \times n$. Upon using the data set (2.3) and letting $\mathbf{y}^{\text {in }} \leftarrow \mathbf{x}(0)$ and $\mathbf{y}^{\text {out }} \leftarrow \mathbf{x}(\Delta)$, the network operator $\mathbf{N}$ can be trained using mean square loss, such that

$$
\mathbf{x}_{m}(\Delta) \approx \mathbf{x}_{m}(0)+\mathbf{N}\left(\mathbf{x}_{m}(0)\right)
$$

where the approximation error is controlled by the training algorithm. Upon satisfactory training of the network, we then obtain a predictive model

$$
\mathbf{x}\left(t_{j+1}\right)=\mathbf{x}\left(t_{j}\right)+\mathbf{N}\left(\mathbf{x}\left(t_{j}\right)\right), \quad j=0, \ldots,
$$

for any arbitrarily given initial condition $\mathbf{x}\left(t_{0}\right)$. Properties of the flow-map based learning were discussed in [9], with its extension for variable time step $\Delta_{j}^{(k)}$ and other system parameters in [7] and systems with missing variables in [2].

2.3. Learning of PDE in Modal Space. In [24, the flow-map based deep learning method was extended to PDE learning. The key is to conduct the learning in modal space, i.e., generalized Fourier space. Consider $u: \Omega \times[0, T] \rightarrow \mathbb{R}, T>0$, as the solution field of an unknown PDE

$$
u_{t}=\mathcal{L}(u) .
$$

One first chooses a set of basis functions in the physical domain $\Omega,\left\{b_{j}(x), j=\right.$ $\left.1, \ldots, N_{b}\right\}$ and expresses the solution as a finite-term series

$$
u(x, t)=\sum_{j=1}^{N_{b}} \hat{u}_{j}(t) b_{j}(x),
$$

where $\hat{\mathbf{u}}(t)=\left[\hat{u}_{1}(t), \ldots, \hat{u}_{N_{b}}(t)\right]^{T}$ are the expansion coefficients. Thus there exists a system of ODEs for the expansion coefficients, in the form of

$$
\frac{d \hat{\mathbf{u}}}{d t}=\mathbf{f}(\hat{\mathbf{u}}) .
$$

If the governing PDE equation is known, such a system for $\hat{\mathbf{u}}$ can be derived via a numerical approximation technique, e.g., Galerkin method. When the governing PDE is unknown, the system for $\hat{\mathbf{u}}$ is unknown as well. When solution data are available, this unknown ODE system can be learned in the modal space by extending flow-map based method from 9. Details of the modal space PDE learning, including its proper mathematical formulation and data processing procedure, can be found in [24]. 
3. DNN Modeling Approach. In this section, we present our DNN modeling of unknown PDE in nodal space. The method allows direct use of measurement data on grid points and does not require structural information of the grids. We first present the basic mathematical formulation, which motivates the base design of our DNN structure. We then discuss the important properties of the proposed DNN network, as well as its extension to learning of system of PDEs and other differential equations.

3.1. Mathematical Motivation. Let $\alpha \in \mathbb{N}_{0}^{d}$ be $d$-variate multi-index with

$$
\alpha=\left(\alpha_{1}, \ldots, \alpha_{d}\right), \quad|\alpha|=\alpha_{1}+\cdots+\alpha_{d} .
$$

Without loss of generality, we consider a $p$-th order autonomous PDE in the following general form,

$$
u_{t}=\mathcal{L}\left(u, \partial^{(1)} u, \ldots \partial^{(p)} u\right), \quad p \geq 1,
$$

where, for any $1 \leq m \leq p$,

$$
\partial^{(m)}=\left\{\partial_{x_{1}}^{\alpha_{1}} \cdots \partial_{x_{d}}^{\alpha_{d}}:|\alpha|=m\right\} .
$$

Note that for multi-dimensional PDE with $d>1$, each $\partial^{(|\alpha|)}$ represents multiple partial derivative operators. Also, $u=\partial^{(0)} u$. Without considering boundary conditions, we assume the PDE (3.1) (1) is well-posed; and (2) has finite number $N_{D} \geq p$ of partial derivative terms.

Let $X_{N}=\left\{x_{1}, \ldots, x_{N}\right\} \subset \Omega$ be the grid set, and $\mathcal{T}=\left\{t^{k}=k \Delta t, k=0, \ldots, K\right\}$ be uniformly distributed (for notational convenience) time instances up to $T=K \Delta t$. We assume that when the PDE (3.1) is known, it can be approximated by a one-step Euler forward type explicit numerical scheme with sufficiently small local truncation error. More specifically, let

$$
\mathbf{v}_{N}^{k+1}=\mathbf{v}_{N}^{k}+\Delta t \cdot \Psi\left(\mathbf{v}_{N}^{k}\right), \quad k=0, \ldots, K-1,
$$

be the numerical scheme, where $\mathbf{v}_{N}^{k}=\left(v\left(x_{1}, t^{k}\right), \ldots, v\left(x_{N}, t^{k}\right)\right)^{T}$ is the numerical solution over $X_{N}$ at time $t^{k}$, and $\boldsymbol{\Psi}$ is the incremental function. We assume that the grid set $X_{N}$ admits a proper spatial discretization of the PDE (3.1) such that

$$
\mathbf{u}_{N}^{k+1}=\mathbf{u}_{N}^{k}+\Delta t \cdot\left(\Psi\left(\mathbf{u}_{N}^{k}\right)+\boldsymbol{\tau}_{N}^{k+1}\right), \quad k=0, \ldots, K-1,
$$

where $\mathbf{u}_{N}^{k}=\left(u\left(x_{1}, t^{k}\right), \ldots, u\left(x_{N}, t^{k}\right)\right)^{T}$ is the exact solution on $X_{N}$ at time $t^{k}$, and $\boldsymbol{\tau}_{N}^{k+1}$ is the local truncation error satisfying

$$
\epsilon\left(X_{N}, \Delta t\right)=\max _{k}\left\|\tau_{N}^{k+1}\left(X_{N}, \Delta t\right)\right\| \ll 1 .
$$

Note that this is a very mild assumption. We only assume that if the PDE (3.1) is known, it can be discretized over the grid set $X_{N}$ and time domain $\mathcal{T}$ with Eulerforward, such that the global truncation error is sufficiently small. The error obviously depends on the choice of spatial discretization over $X_{N}$ and scales as $O(\Delta t)$ because of Euler-forward temporal discretization. We do not assume the stability of the scheme.

Proposition 3.1. Under the assumption that the PDE (3.1) admits the Euler forward explicit numerical method (3.2) satisfying (3.4), there exists a set of functions $\left\{F_{i}: \mathbb{R}^{N} \rightarrow \mathbb{R}^{N}, i=1, \ldots, J\right\}, J \geq 1$, and an iterative scheme

$$
\mathbf{v}_{N}^{k+1}=\mathbf{v}_{N}^{k}+\Delta t \cdot \mathcal{M}\left[F_{1}\left(\mathbf{v}_{N}^{k}\right), \cdots, F_{J}\left(\mathbf{v}_{N}^{k}\right)\right], \quad k=0, \ldots, K-1,
$$


where $\mathcal{M}$ is a (nonlinear) function operated component-by-component, such that for sufficiently large $J$, the exact solution of (3.1) satisfies

$$
\mathbf{u}_{N}^{k+1}=\mathbf{u}_{N}^{k}+\Delta t \cdot\left(\mathcal{M}\left[F_{1}\left(\mathbf{u}_{N}^{k}\right), \cdots, F_{J}\left(\mathbf{u}_{N}^{k}\right)\right]+\boldsymbol{\eta}_{N}^{k+1}\right), \quad k=0, \ldots, K-1,
$$

where $\left\|\boldsymbol{\eta}_{N}^{k+1}\right\| \leq \epsilon\left(X_{N}, \Delta t\right)$, the global truncation error defined in (3.4), $\forall k$.

Proof. The existence of (3.5) can be proved by construction, based on the explicit Euler-forward scheme (3.2). Given the grid set $X_{N}$, there exist linear operators to approximate partial derivatives. More specifically, consider a sufficiently smooth function $w(x)$ and its grid function $\left.w(x)\right|_{X_{N}}=\left(w\left(x_{1}\right), \ldots, w\left(x_{N}\right)\right)^{T}:=\mathbf{w}$. Then, its partial derivatives on $X_{N}$ can be approximated via differentiation matrices. That is, for any $\alpha$ with $1 \leq|\alpha| \leq p$, there exists a differentiation matrix $\mathbf{D}^{\alpha}: \mathbb{R}^{N} \rightarrow \mathbb{R}^{N}$ such that,

$$
\left.\partial_{x_{1}}^{\alpha_{1}} \cdots \partial_{x_{d}}^{\alpha_{d}} w(x)\right|_{X_{N}} \approx \mathbf{D}^{\alpha} \mathbf{w}
$$

Upon using Euler forward time stepping over $\mathcal{T}$, one can construct the following explicit numerical approximation to the PDE (3.1).

$$
\mathbf{v}_{N}^{k+1}=\mathbf{v}_{N}^{k}+\Delta t \cdot \mathcal{L}\left(\mathbf{v}_{N}^{k}, \mathbf{D}^{(1)} \mathbf{v}_{N}^{k}, \cdots, \mathbf{D}^{(p)} \mathbf{v}_{N}^{k}\right), \quad k=0, \ldots, K-1,
$$

where

$$
\mathbf{D}^{(m)}=\left\{\mathbf{D}^{\alpha}:|\alpha|=m\right\}, \quad 1 \leq m \leq p .
$$

This is a special case of (3.5), where $F_{1}$ is the identity operator, $F_{2}, \ldots, F_{J}$ correspond to the differentiation matrices in (3.8), and $J=N_{D}+1$. Note $N_{D}$ is the number of partial derivatives in (3.1). The existence of (3.5) is thus established.

REMARK 3.1. Constructing differentiation matrices over a grid set $X_{N}$, in the form of (3.7), is a standard procedure in collocation type numerical PDE methods, e.g., finite difference method, spectral collocation method, radial basis method, etc. The accuracy of the differentiation matrix approach depends primarily on the property of the grid $X_{N}$, the chosen approximation approach, and the regularity of the function under consideration.

REMARK 3.2. The accuracy of the Euler forward linear approximation scheme (3.8), manifested by its truncation error (3.4), is obviously limited. In particular, its temporal accuracy is limited to first order $O(\Delta t)$. However, the scheme (3.8) is only needed to establish the existence of (3.5), which is of more general nonlinear form. The nonlinear scheme (3.5) serves as the foundation of our deep learning algorithm, which will not be restricted to by the accuracy of the Euler forward method (3.8).

3.2. Basic DNN Structure. Deep neural networks (DNNs) are naturally nonlinear approximators. In this section, we discuss our DNN structure design to achieve the nonlinear approximation, in the form of (3.5), of an unknown PDE (3.1). Once successfully trained via the available data set (2.2), the DNN model provides an iterative scheme, as in (3.5), that can be used as a predictive model of the underlying unknown PDE.

The basic structure of our DNN model is illustrated in Figure 3.1. It consists of the following components:

- Input layer, where the number of neurons is $N$, the dimensionality of $\mathbf{u}$. 


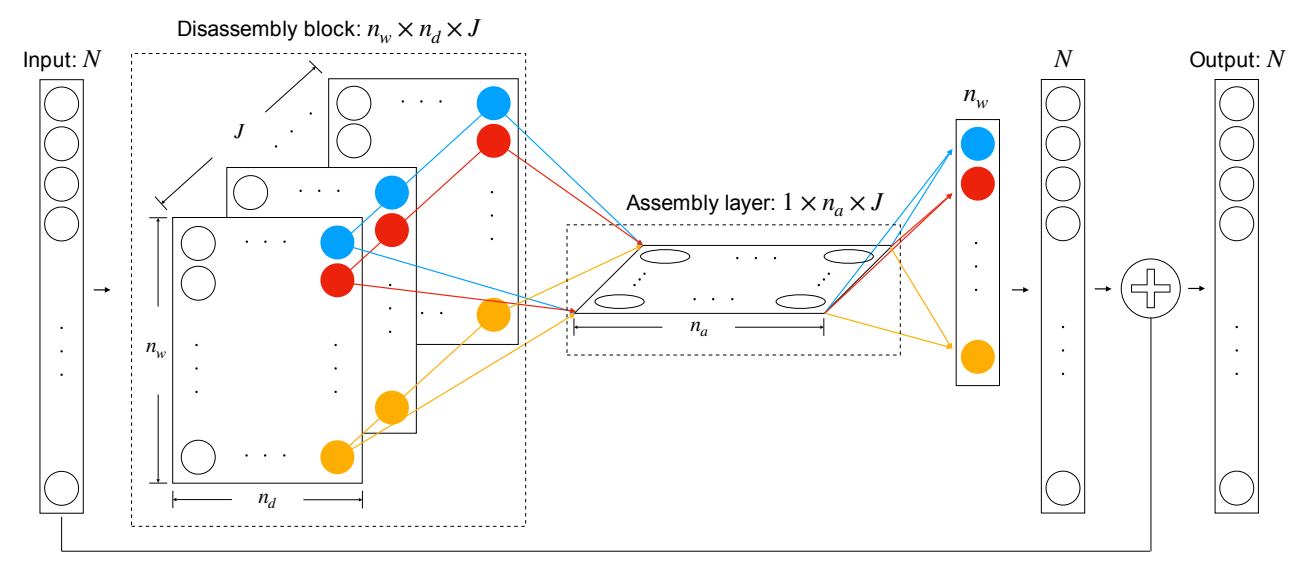

Fig. 3.1: The basic DNN structure.

- Disassembly block. It has $J \geq 1$ fully connected FNNs "in parallel", where each FNN has width $n_{w}$ and depth $n_{d}$ and receives inputs from the input layer. As illustrated in Figure 3.1, the disassembly block thus creates a 3dimensional tensor structure with dimension $n_{w} \times n_{d} \times J$, where $J$ shall be referred to as the "thickness" of the disassembly block hereafter. The output layers of the disassembly block create a matrix structure of $n_{w} \times J$, spanning the width and thickness directions of the block.

- Assembly layer. It is a standard fully connected FNN, with width $J$ and depth $n_{a}$. It operates along the thickness direction of the disassembly block. It receives input from one of the "rows" of the disassembly block output matrix, whose size is $n_{w} \times J$, and produces a scalar output. This is repeated for each of the $n_{w}$ rows of the disassembly output matrix and produces an output vector of $n_{w}$. (In other words, one can also view the assembly layer as a block of $n_{w}$ identical FNNs, i.e., with shared parameters, stacked vertically.)

- Output layer, where the number of neurons is $N$, the dimensionality of $\mathbf{u}$. The output layer is mapped from the output of the assembly layer, whose dimension is $n_{w}$. The input layer is re-introduced before the final output, in the same manner of residual network (ResNet).

Let $\mathcal{N}_{i}, i=1, \ldots, J$, be the operators defined by the parallel FNNs inside the disassembly block, i.e.,

$$
\mathcal{N}_{i}: \mathbb{R}^{N} \rightarrow \mathbb{R}^{n_{w}}, \quad i=1, \ldots, J .
$$

Let $\mathcal{D}$ denote the operator defined by the disassembly block. We then have, with a slight abuse of notation,

$$
\mathcal{D}=\bigotimes_{1 \leq i \leq J} \mathcal{N}_{i}: \mathbb{R}^{N} \rightarrow \mathbb{R}^{n_{w} \times J}
$$

Let $\mathbf{A}$ be the operator defined by the assembly layer. We have

$$
\mathbf{A}: \mathbb{R}^{J} \rightarrow \mathbb{R}
$$


which is applied to each "row" of the output of the disassembly block. The "componentwise" application of $\mathbf{A}$ results in the following operator composition:

$$
\mathbf{A} \circ \mathcal{D}: \mathbb{R}^{N} \rightarrow \mathbb{R}^{n_{w}} .
$$

Let

$$
\mathcal{F}: \mathbb{R}^{n_{w}} \rightarrow \mathbb{R}^{N}
$$

be an operator mapping between $n_{w}$-vector and $N$-vector. We can then write the operator for the entire network structure, shown in Figure 3.1 as

$$
\mathcal{N}=\mathbf{I}_{N}+\mathcal{F} \circ \mathbf{A} \circ \mathcal{D}: \mathbb{R}^{N} \rightarrow \mathbb{R}^{N},
$$

where $\mathbf{I}_{N}$ is the identity matrix of $N \times N$. The proposed neural network thus defines the following operation: for $\mathbf{w}^{\text {in }} \in \mathbb{R}^{N}$ and $\mathbf{w}^{\text {out }} \in \mathbb{R}^{N}$,

$$
\mathbf{w}^{\text {out }}=\mathcal{N}\left(\mathbf{w}^{\text {in }} ; \Theta\right)=\mathbf{w}^{\text {in }}+\mathcal{F}\left[\mathbf{A}\left(\mathcal{N}_{1}\left(\mathbf{w}^{i n}\right), \ldots, \mathcal{N}_{J}\left(\mathbf{w}^{i n}\right)\right)\right],
$$

where $\Theta$ is the set of network parameters and $\mathbf{A}$ is operated component-wise.

3.3. Model Properties. Let us first consider a special case of the proposed DNN model, where the width of each of the FNN in the disassembly block $n_{w}=N$, the dimensionality of the input. In this case, the output of the assembly layer also has dimension $N$, i.e., from (3.12) we have

$$
\mathbf{A} \circ \mathcal{D}: \mathbb{R}^{N} \rightarrow \mathbb{R}^{N}
$$

Subsequently, the $\mathcal{F}$ operator 3.13 can take the form of the identity operator $\mathbf{I}_{N}$. The corresponding DNN model (3.15) becomes

$$
\mathbf{w}^{\text {out }}=\mathbf{w}^{\text {in }}+\mathbf{A}\left(\mathcal{N}_{1}\left(\mathbf{w}^{i n}\right), \ldots, \mathcal{N}_{J}\left(\mathbf{w}^{\text {in }}\right)\right)
$$

This is a direct correspondence to the mathematical model 3.5 in Proposition 3.1 Each FNN in the disassembly block approximates the $F$ 's function in (3.5),

$$
\mathcal{N}_{i} \approx F_{i}, \quad i=1, \ldots, J
$$

and the assembly layer approximates the $\mathcal{M}$ operator in (3.5),

$$
\mathbf{A} \approx \mathcal{M}
$$

Courtesy of the universal approximation property of neural networks (cf. [16]), the DNN model (3.17) thus can approximate the general mathematical model (3.5) arbitrarily well, when all the FNNs involved in the network have sufficient complexity.

The general case of $n_{w} \neq N$ is worth discussing, especially the case of $n_{w}<N$. In many problems, the dimensionality of the grid set $X_{N}$ can be exceedingly large, i.e., $N \gg 1$. Adopting $n_{w}<N$, and in some cases, $n_{w} \ll N$, can be computationally advantageous. In this case, the core of the proposed DNN model, which consists of the disassembly block and assembly layer, performs (3.12) and achieves effectively a model reduction operation $\mathbb{R}^{N} \rightarrow \mathbb{R}^{n_{w}}$. Subsequently, the $\mathcal{F}$ operator (3.13) achieves a "lifting" operation back to the higher dimension spacer $\mathbb{R}^{n_{w}} \rightarrow \mathbb{R}^{N}$. This inherent model reduction capability of our DNN model is worth noting. This property and its 
analysis are beyond the scope of this paper and will be pursued in a separate study. In this paper, we will focus on the special case of $n_{w}=N$.

Another notable property of our DNN model is that both the input vector $\mathbf{w}^{\text {in }}$ and output vector $\mathbf{w}^{\text {out }}$ can be arbitrarily permuted, so long as they are subjected to the same permutation. Since the indexing of these vectors follow the grid set $X_{N}$, an immediate consequence is that the DNN model requires no geometric information of the underlying grid set $X_{N}$. Consequently, our DNN model can work with arbitrarily distributed grid $X_{N}$ and results in a mesh-free predictive model.

3.4. Model Training and Prediction. Our nodal PDE learning model is constructed by training the DNN model (3.15) with the data set (2.2). Upon setting

$$
\mathbf{w}^{i n} \leftarrow \mathbf{u}_{j}(0), \quad j=1, \ldots, M,
$$

we minimize the mean squared loss

$$
L(\Theta ; \Delta t)=\frac{1}{M} \sum_{j=1}^{M}\left\|\mathcal{N}\left(\mathbf{u}_{j}(0) ; \Theta\right)-\mathbf{u}_{j}(\Delta t)\right\|_{2}^{2}
$$

to obtain the network hyperparameters $\Theta$.

Once trained, we obtain a predictive model over the grid set $X_{N}$ for the underlying unknown PDE. For an arbitrarily given initial condition $\mathbf{u}_{N}(0)$ over the grid $X_{N}$, we have

$$
\left\{\begin{array}{l}
\mathbf{v}_{N}^{0}=\mathbf{u}_{N}(0), \\
\mathbf{v}_{N}^{k+1}=\mathcal{N}\left(\mathbf{v}^{k}\right)=\mathbf{v}_{N}^{k}+\mathcal{F}\left[\mathbf{A}\left(\mathcal{N}_{1}\left(\mathbf{v}_{N}^{k}\right), \ldots, \mathcal{N}_{J}\left(\mathbf{v}_{N}^{k}\right)\right)\right], \quad k=0, \ldots
\end{array}\right.
$$

Although this DNN model resembles the Euler forward type of numerical methods, it is fundamentally different. The traditional numerical methods, including Euler forward, are defined through predetermined coefficients and parameters. They are usually linear methods and applicable to general PDEs. The DNN model (3.22) is fully nonlinear, in the sense that it is constructed specifically for the PDE system behind the training data. As a result, the DNN model does not have temporal error associated with $\Delta t$, as in the traditional numerical methods (cf. [9]). On the other hand, the trained DNN model can only be applied to prediction of the PDE associated with the training data and not to another PDE system.

Numerical stability of the predictive model (3.22) can be not fully analyzed at the moment, due to the lack of mathematical tools for DNNs. However, we have discovered that, upon extensive numerical experimentation, numerical stability of the DNN model can be drastically improved by using recurrent loss, i.e., loss computed over multiple time steps. Let $n_{L} \geq 1$ be the number of time steps for loss computation, our loss function is defined as

$$
L(\Theta)=\sum_{n=1}^{n_{L}} L(\Theta ; n \Delta t)
$$

where, for $n=1, \ldots, n_{L}$,

$$
L(\Theta ; n \Delta t)=\frac{1}{M} \sum_{j=1}^{M}\left\|\mathcal{N}^{n}\left(\mathbf{u}_{j}(0) ; \Theta\right)-\mathbf{u}_{j}(n \Delta t)\right\|_{2}^{2},
$$


with $\mathcal{N}^{n}=\mathcal{N} \circ \cdots \circ \mathcal{N}$ composed $n$ times. Note that the mean squared loss defined in (3.21) is merely a specical case of $n_{L}=1$. The use of the recurrent loss with $n_{L}>1$ does require us to re-orgainize the training data set (2.2). In this case, the training data set needs to contain data sequences of length $n_{L}+1$.

$$
\left(\mathbf{u}_{m}(0), \mathbf{u}_{m}(\Delta t), \ldots, \mathbf{u}_{m}\left(n_{L} \Delta t\right)\right), \quad m=1, \ldots, M .
$$

Again, the original data set (2.2) is a special case of $n_{L}=1$.

\subsection{Extensions.}

3.5.1. PDE Systems. The proposed DNN model can be extended to system of PDEs in a straightforward manner. For a system of PDEs with state variables $U=\left(u_{1}, \ldots, u_{L}\right)$ and governed by unknown PDE system

$$
\partial_{t} u_{i}=\mathcal{L}_{i}(U), \quad i=1, \ldots, L,
$$

where the PDE operator $\mathcal{L}_{i}$ includes partial derivatives of $U$. Our DNN model (3.15) for scalar PDE (3.1), as illustrated in Figure 3.1 can be extended to the system by merely concatenating all the state variables into a single larger vector. That is, let $\mathbf{u}_{i} \in \mathbb{R}^{N}$ be the data snapshot of each component $u_{i}, i=1, \ldots, L$. We then define

$$
\mathbf{U}=\left[\mathbf{u}_{1}^{T}, \cdots, \mathbf{u}_{L}^{T}\right]^{T} \in \mathbb{R}^{N L}
$$

as the concatenated state vector. The DNN structure and network model in the previous sections can then be applied directly to $\mathbf{U}$.

Note that it is possible, and maybe even preferable mathematically, to construct a more "refined" DNN structure to apply to each of the PDEs in the system. However, our early numerical experimentation indicated that the straightforward concatenation approach is a flexible numerical approach. Further refinement in DNN structure for PDE systems will be investigated in a future study.

3.5.2. Other Differential-type Equations. The proposed DNN structure in Section 3.2 has its foundation in Section (3.1), particularly in Proposition 3.1. The key ingredient is that partial derivatives can be approximated by the solution values over the grid $X_{N}$. This is explicitly achieved in the standard numerical methods via techniques such as differentiation matrices; and implicitly achieved in our DNN model via the disassembly block structure. A natural extension is that operators other than partial differentiation can also be incorporated and modeled by the same DNN structure, as long as the operator can be approximated using solution values over the grid $X_{N}$. One obvious example is integral operator over the domain $\Omega$. More specifically, one can always use data over the grid $X_{N}$ to approximate an integral, i.e.,

$$
\int_{\Omega} u(x) d x \approx G\left(u\left(x_{1}\right), \ldots u\left(x_{N}\right)\right) .
$$

The existence of $G$ is obvious, as there are a variety of integration rules (e.g, quadrature, sampling methods) to accomplish the approximation. Therefore, if such an integral exists in the PDE, the same DNN structure in Section 3.2 can be applied. The applicability of the proposed DNN model to differential-integral equations will be demonstrated in our numerical example section. 
4. Computational Studies. In this section, we present numerical examples to demonstrate the properties of the proposed DNN modeling method for PDEs. In all of the examples, the true governing PDEs are known. These true PDEs serve only two purposes: (1) to generate synthetic training data. This is achieved by solving the true PDEs either analytically (if possible) or by high resolution numerical solvers (most of the cases). Once the training data sets are generated, they are used to train DNN models for the PDEs behind the data; and (2) to generate reference solutions for validating the DNN model predictions. Therefore, the knowledge of the true PDEs does not in any way facilitate the DNN model construction. Also, the training data sets (3.24) consist of very short trajectories of length $n_{L} \times \Delta t$, where $n_{L}$ is the number of time steps in computing the loss function. In all of the examples, we have $n_{L} \leq 10$. For predictions, we typically march the DNN models under arbitrarily given new initial conditions that are not in the training data set for a much longer time horizon. Therefore, all our DNN model predictions are "out-of-sample".

The one-dimensional examples have periodic boundary conditions, and the twodimensional example has Dirichlet boundary conditions. Note that the prescribed boundary conditions are used only to generate the training data. The boundary conditions are not enforced in the DNN modeling structure. Instead, the DNN models "learn" the correct boundary conditions embedded in the training data and proceed to produce predictions over longer time.

For the one-dimensional examples with periodic boundary conditions, we generate training data by solving the true PDEs with the following "initial conditions", in the form of finite Fourier series,

$$
u(x, 0)=a_{0}+\sum_{n=1}^{N_{c}}\left(a_{n} \cos (n x)+b_{n} \sin (n x)\right),
$$

where the Fourier coefficients $\left\{a_{n}, b_{n}\right\}$ are randomized to generate different initial conditions. The training data sets are obtained by solving the true PDEs for $n_{L}$ steps, as shown in (3.23), from these initial conditions. In most of our examples, we set $n_{L}=10$.

Our numerical experimentations, along with the earlier work on flow map based learning ( $9,7,24,2$, indicate that one does not need exceedingly complex DNNs for accurate modeling. Therefore, in the following examples, the disassembly block and assembly layer have depths $n_{d}=1$ and $n_{a}=1$, while the width and thickness of the disassembly block are set as $n_{w}=N$ and $J=5$. Hence, unless otherwise noted, our DNNs have disassembly block of dimension (width $\times$ depth $\times$ thickness) $N \times 1 \times 5$, and assembly layer of dimension $1 \times 1 \times 5$, as illustrated in Fig. 3.1.

4.1. Advection-Diffusion Problem. We first consider an advection-diffusion equation with $2 \pi$-periodic boundary condition,

$$
\frac{\partial u(x, t)}{\partial x}+\frac{\partial}{\partial x}(\alpha(x) u(x, t))=\frac{\partial}{\partial x}\left(\kappa(x) \frac{\partial u(x, t)}{\partial x}\right),
$$

where $\alpha$ is transport velocity and $\kappa$ is diffusivity. Note that both $\alpha$ and $\kappa$ are functions. Without loss of generality, we set the functions as deterministic functions, in the Fourier series form (4.1), with (arbitrarily chosen) fixed coefficients listed in Table 4.1 . Upon setting up the true governing equation, we use it to generate training data. The data are generated by solving the equation with a high-resolution numerical solver Fourier collocation in space and Crank-Nicolson in time. We employ 10,000 training 
data trajectories, each of which are generated by solving the true equation with a randomized initial condition (4.1) with $a_{0}=0, a_{n}$ and $b_{n}$ drawn from a continuous uniform distribution over $[-1,1]$, i.e. $a_{n}, b_{n} \in U[-1,1]$, and $N_{c}$ chosen from a discrete uniform distribution over $\{1, \ldots, 10\}$, i.e. $N_{c} \in U\{1, \ldots, 10\}$. The training trajectory data have time step $\Delta t=0.02$ and length $n_{L}=10$ in the loss function (3.23). This implies that our training data are trajectories of length only $t=0.2$.

\begin{tabular}{|c|c|c|}
\hline$\alpha(x)$ & $a_{0}$ & 1 \\
& $a_{n}$ & $(0.5426,0.2673,-0.0030,-0.6039,0.6618) \times 0.05$ \\
& $b_{n}$ & $(-0.9585,0.4976,-0.5504,0.5211,-0.8233) \times 0.05$ \\
\hline$\kappa(x)$ & $a_{0}$ & $1 \times 10^{-3}$ \\
& $a_{n}$ & $(0.3707,-0.9921,0.62524,0.4435,0.8355) \times 5 \times 10^{-5}$ \\
& $b_{n}$ & $(0.9068,0.0243,0.2251,-0.4162,0.4291) \times 5 \times 10^{-5}$ \\
\hline
\end{tabular}

Table 4.1: The fixed parameters for $\alpha(x)$ and $\kappa(x)$ in (4.2), in the form of (4.1).

Hyperbolic tangent function "tanh" is used as the activation function throughout. A cyclic learning rate is used during training, with maximum learning rate of $1 \times 10^{-3}$, minimum learning rate of $1 \times 10^{-4}$, and exponential decay rate of 0.99994 . We trained the DNN for 5,000 epochs with batch size 50 .

We first consider DNN modeling on uniform grids in physical space. In particular, we consider the nodes $x_{i}=\frac{2 \pi}{N} i, i=0, \ldots, N-1$, where $N=50$. To validate the trained DNN model, we conduct numerical predictions with initial conditions not in the training data set. Here, we present the results by an initial condition $u(x, 0)=$ $\exp \left(-\sin ^{2}(x)\right)-1 / 2$. In Fig. 4.1, the DNN predictions are plotted at different time instances, along with the reference solutions (by solving the true model using the same initial condition) for comparison. We observe that the DNN model produces accurate prediction results for up to $t=20$, which is a much longer time span than the training data set (for which $t=0.2$ ).

We then consider DNN modeling on an unstructured grid. Specifically, we randomly perturb the uniform grid from above by up to $\pm 25 \%$ of the interval size. We then randomly permute the grid ordering. The resulting grids are therefore void of any geometric information. We then train the DNN on this randomized grid set. The DNN predictions using the initial condition $u(x, 0)=\exp \left(-\sin ^{2}(x)\right)-1 / 2$ are shown in Fig. 4.2. We again observe good agreement with the reference solution for time up to $t=20$. This demonstrates the flexibility of the proposed DNN modeling, which can be completely structure-free.

4.2. 4th-order PDE. We now consider a 4th-order PDE,

$$
\frac{\partial u}{\partial t}+c \frac{\partial^{4} u}{\partial x^{4}}=0
$$

with $c=1 \times 10^{-2}$ and $2 \pi$-periodic boundary condition.

We consider a uniform grid with nodes given by $x_{i}=\frac{2 \pi}{N} i, i=0, \ldots, N-1$, with $N=50$. The training data are generated using the Fourier series (4.1), with $N_{c}=$ 7 and uniformly distributed random coefficients $a_{0} \sim[-1 / 2,1 / 2]$, and $a_{n}^{(i)}, b_{n}^{(i)} \sim$ $U\left[-\frac{1}{n}, \frac{1}{n}\right], 1 \leq n \leq N_{c}$. The time step is $\Delta t=1 \times 10^{-2}$, and the length of the training trajectories is $n_{L}=10$, as in (3.23). The training data set consists of 10,000 such trajectories. The tanh activation function is used throughout, along with cyclic 

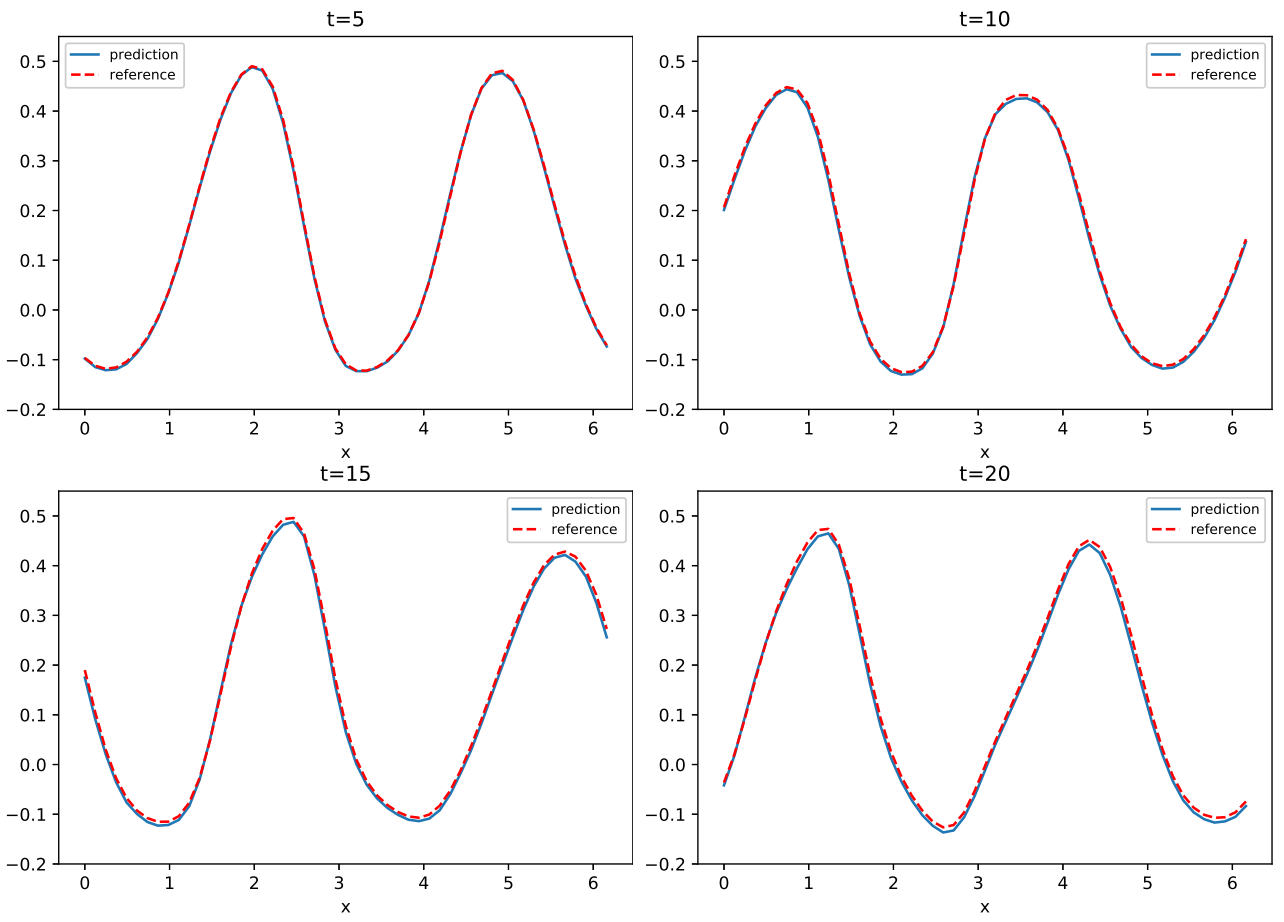

Fig. 4.1: Advection-diffusion on uniform grids. Comparison between DNN model prediction and reference solution.

learning with maximum learning rate of $1 \times 10^{-3}$, minimum learning rate of $1 \times 10^{-4}$, and exponential decay rate 0.99994 . The DNN model is trained for 2, 000 epochs with batch size 50 .

Validation results with the initial condition $u(x, 0)=\exp \left(-\sin ^{2}(x)\right)-1 / 2$ are shown in Fig. 4.3 We observe excellent agreement between the DNN model predictions and the reference solutions over time.

4.3. Differential-Integral Equation. As discussed in Section 3.5.2, the proposed DNN structure can be used to model other types of equations involving operators such as integrals, see (3.27). Here, we consider a differential-integral equation,

$$
\frac{\partial u}{\partial t}+\frac{\partial u}{\partial x}=\frac{1}{2 \pi} \int_{0}^{2 \pi} u(y, t) d y-u
$$

with $2 \pi$-periodic boundary condition.

The training data are generated on a uniform grid with nodes given by $x_{i}=\frac{2 \pi}{N} i$, $i=0, \ldots, N-1$ with $N=50$. A total number of 10,000 trajectories with time step $\Delta t=5 \times 10^{-4}$ are stored over $n_{L}=10$ steps (3.23). Note that the training trajectory data are of very short temporal length, only up to $t=0.005$. The trajectories are generated by solving the true equation with the Fourier initial condition (4.1) with $a_{0}=0, a_{n}, b_{n} \sim U[-1,1]$, and $N_{c} \sim U\{0, \ldots, 10\}$.

The hyperbolic tangent function tanh is used as the activation function, along with cyclic learning with maximum learning rate of $1 \times 10^{-3}$, minimum learning rate 

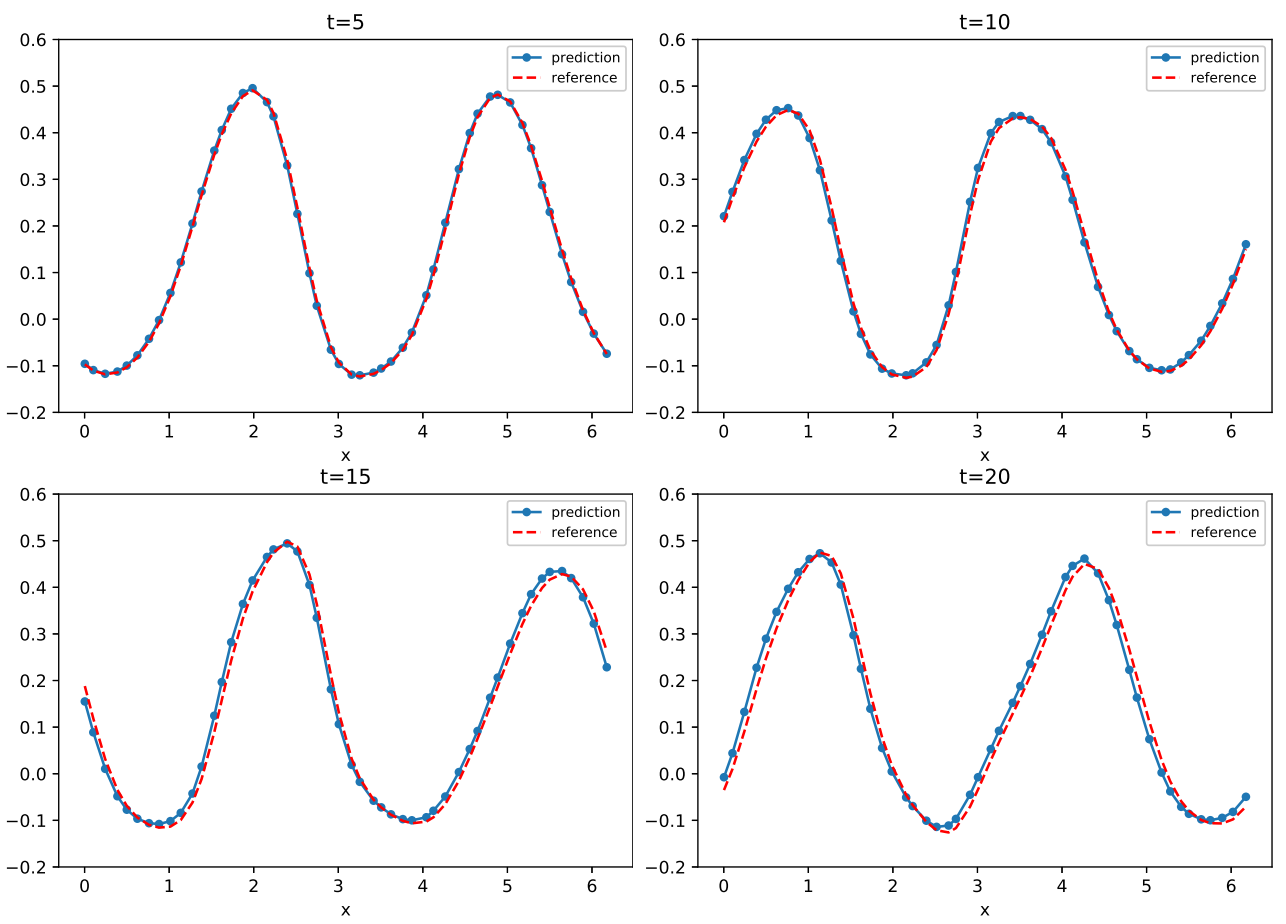

Fig. 4.2: Advection-diffusion on unstructured grids. Comparison between DNN model prediction and reference solution. The non-uniform grids are the solid dots, whose indices are randomly permuted.

of $1 \times 10^{-4}$, and exponential decay rate of 0.99994 . The DNN model was trained for 30, 000 epochs with batch size 50 .

In Fig. 4.4 the validation results obtained using the initial condition

$$
u(x, 0)=\exp \left(-\frac{1}{5} \sin (3 x)\right)-\frac{1}{2} \cos (x)-1
$$

are shown. We observe excellent agreement with the reference solution, obtained by solving the true equation. The results are up to $t=1.5$, which is significantly longer than the temporal length of the training data (for which $t=0.005$ ).

4.4. Burger's Equation. In this section we consider Burger's equation, in both viscous and inviscid form. The latter case poses significant numerical challenges, as shocks may develop in finite time.

4.4.1. Viscous Burger's Equation. We first consider viscous Burgers equation

$$
\frac{\partial u}{\partial t}+u \frac{\partial u}{\partial x}=\nu \frac{\partial^{2} u}{\partial x^{2}}
$$

with $2 \pi$-periodic boundary condition and viscosity $\nu=0.1$.

Similar to the other cases, the training data are generated by solving the true equation (4.5) on a uniform grid with nodes given by $x_{i}=-\pi+\frac{2 \pi}{N} i, i=0, \ldots, N-$ 

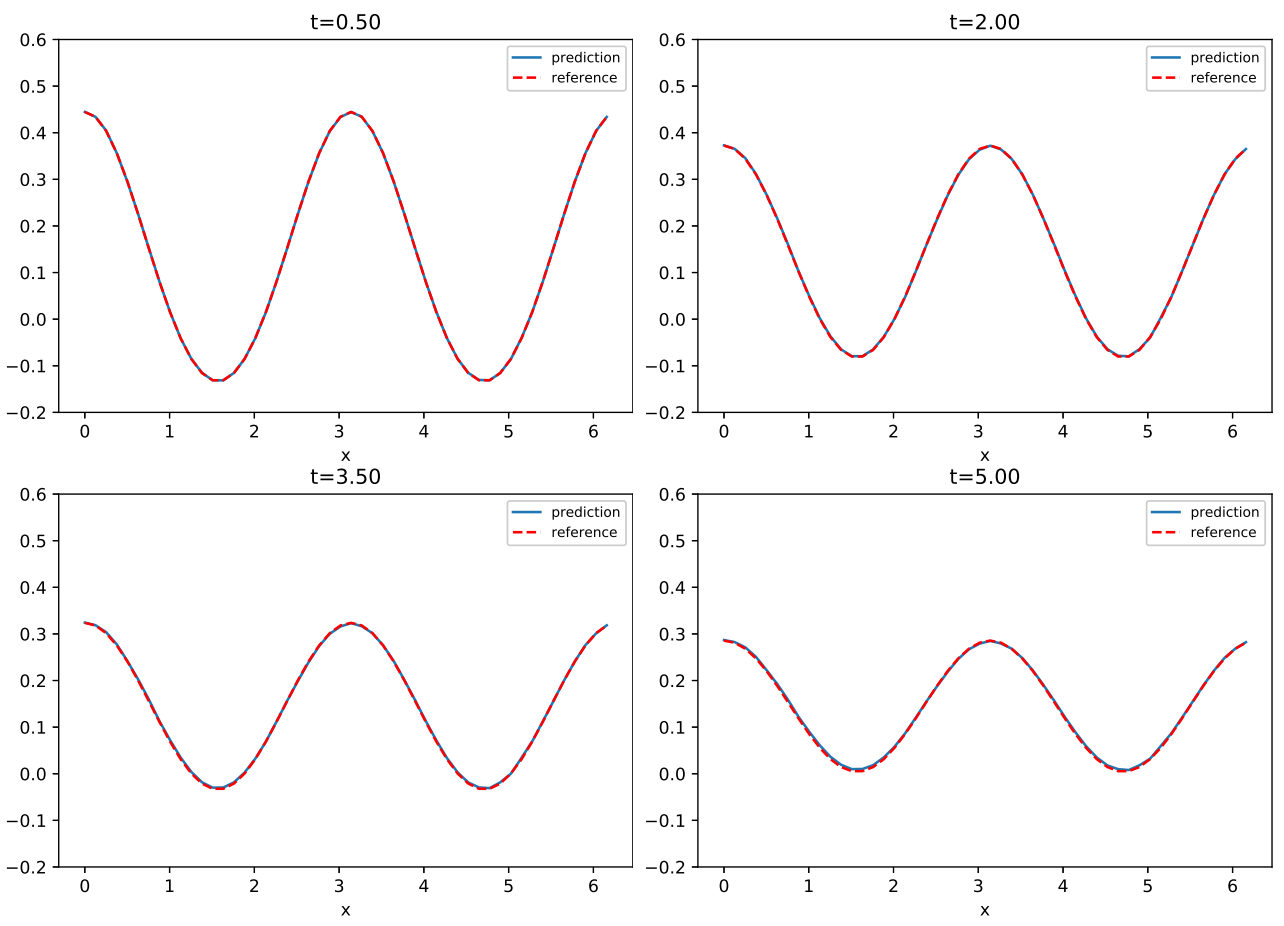

Fig. 4.3: 4th-order PDE: Comparison between DNN model prediction and reference solution.

1 where $N=50$. The Fourier series (4.1) is used to generate 10,000 randomized trajectory data using coefficients $a_{0} \sim U[-1 / 2,1 / 2]$ and $a_{n}, b_{n} \sim U\left[-\frac{1}{n}, \frac{1}{n}\right]$ for $1 \leq$ $n \leq N_{c}$, and $N_{c}=10$. The trajectories are of length $n_{L}=10$ and with time step $\Delta t=0.01$, for the multi-step loss computation (3.23).

Cyclic learning is used, with maximum learning rate $1 \times 10^{-3}$, minimum learning rate $1 \times 10^{-4}$, and an exponential decay rate 0.99994 , along with tanh as the activation function. The DNN was trained for 20, 000 epochs with batch size 50 .

In Fig. 4.5, we present the DNN prediction under initial condition $u(x, 0)=$ $\sin (x)$, along with the reference solution. We observe that the DNN generates an accurate prediction up to $t=5$. Note that the solution starts to develop a sharper slope early on, before the viscous term takes effect and diffuses it away. Without the viscous term, a shock will develop, as shown in the next example.

4.4.2. Inviscid Burger's Equation. We now consider inviscid Burgers equation with $2 \pi$-periodic boundary condition,

$$
\frac{\partial u}{\partial t}+\frac{\partial}{\partial x}\left(\frac{1}{2} u^{2}\right)=0
$$

The training data set for this example contains 20,000 trajectory data. The first 10,000 are generated in the same manner as in the viscous Burger's case in the previous section. In addition, we include 10,000 discontinuous solution data, which 

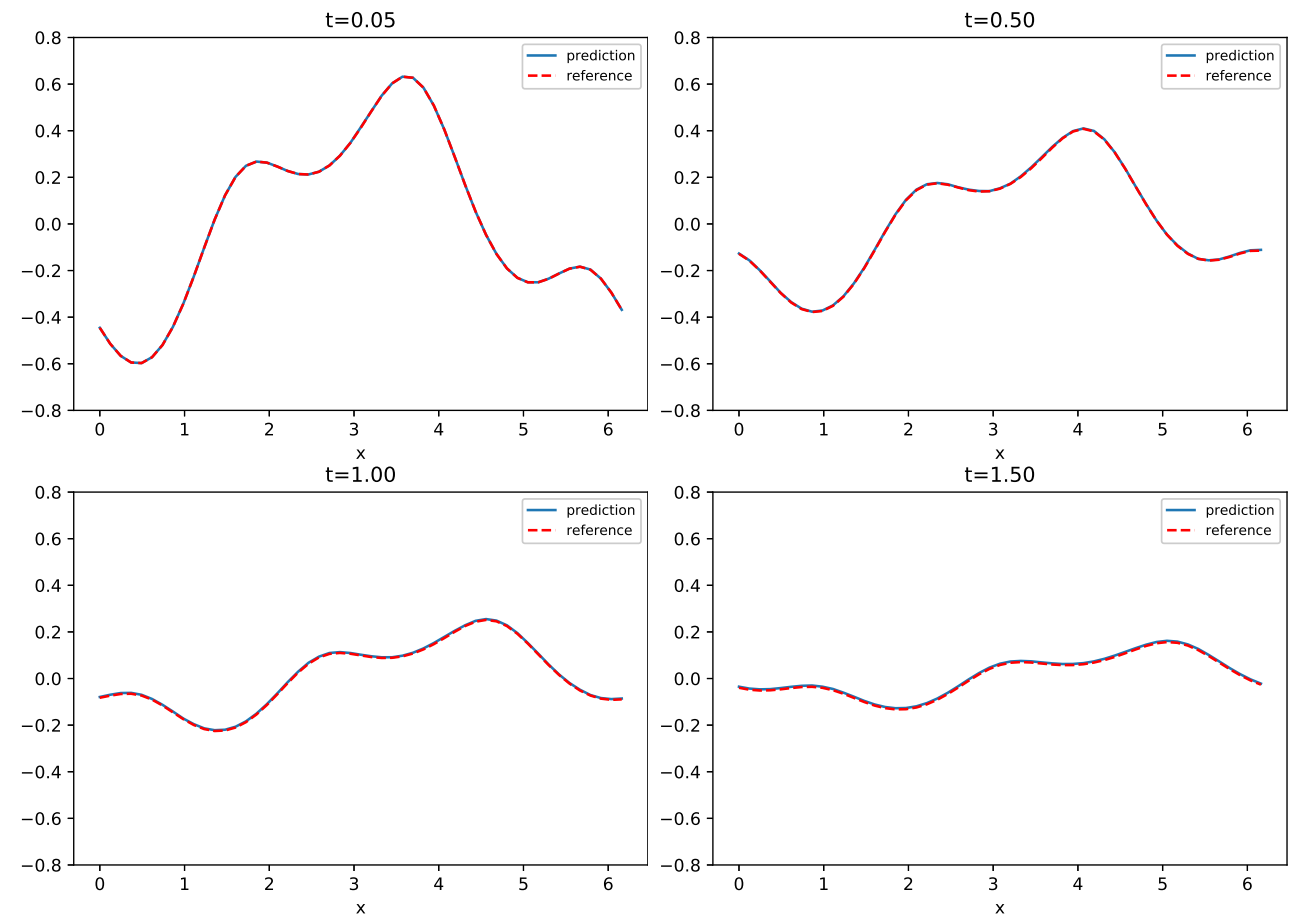

Fig. 4.4: Differential-integral equation: Comparison between DNN model prediction and reference solution.

are generated from randomized piecewise constant initial data

$$
u_{0}(x)=\left\{\begin{array}{lc}
u_{1}, & x_{\ell} \leq x \leq x_{r} \\
u_{2}, & \text { elsewhere }
\end{array}\right.
$$

where $u_{1}, u_{2} \sim U[-1,1]$ and $x_{\ell}, x_{r} \sim U[-\pi, \pi]$. For each of these piecewise constant initial conditions, we generated $n_{L}=10$ steps of training trajectory data with time step $\Delta t=0.01$, by numerically solving the true equation using 9th-order WENO method in space and 4th-order Runge-Kutta method in time.

The DNN structure and its training are set in the same manner as in the viscous Burger's example in the previous section. The prediction results generated by the trained DNN model using the initial condition $u(x, 0)=\sin (x)$ are shown in Fig. 4.6. In this case, the solution develops a shock discontinuity at time $t=2$, which the DNN model correctly predicts. In particular, the predicted solution becomes sharper as time marches. At $t=1.5$, when the solution becomes very sharp near $x=0$, the DNN prediction exhibits small oscillations. This is a common feature of numerical solutions for sharp transition layers. At $t=2$, the shock is fully developed (as shown by the reference solution). The DNN prediction accurately captures the shock location and solution profile. Again, small numerical oscillations are visible near the shock location, as is common in most numerical solutions. 

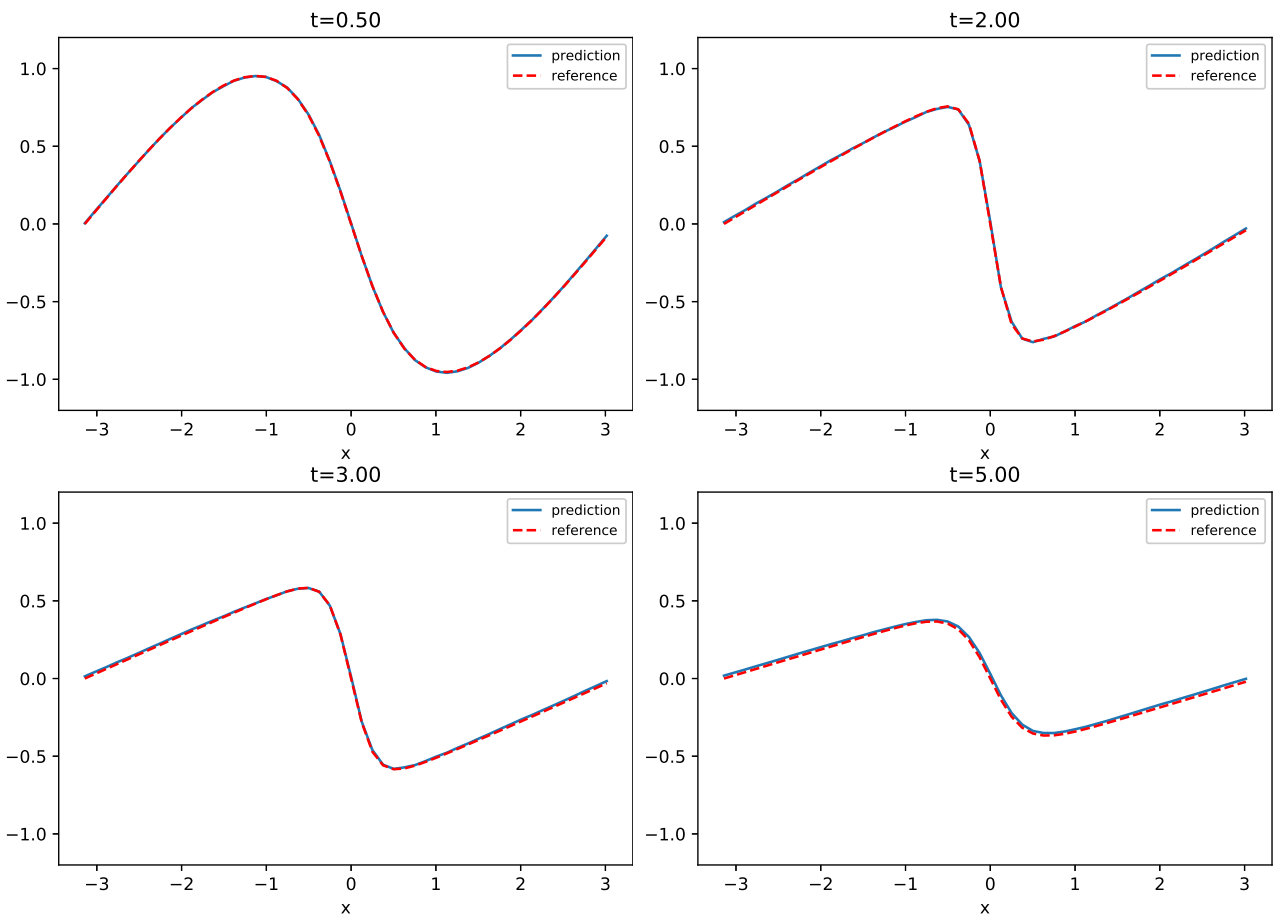

Fig. 4.5: Viscous Burger's equation with viscosity $\nu=0.1$. Comparison of DNN model prediction and reference solution.

4.5. PDE System. We now discuss the proposed DNN learning for systems of PDEs. For benchmarking purposes we consider a linear wave system

$$
\mathbf{u}_{t}=\mathbf{A} \mathbf{u}_{x}
$$

where

$$
\mathbf{u}=\left[\begin{array}{l}
u_{1}(x, t) \\
u_{2}(x, t)
\end{array}\right] \text { and } \quad \mathbf{A}=\left[\begin{array}{ll}
0 & 1 \\
1 & 0
\end{array}\right]
$$

with $2 \pi$-period boundary condition. (Note that even though its form is simple, linear wave equations like this are exceptionally difficult to solve accurately using numerical methods.)

The training data are collected over a uniform grid with $N=50$ points and $n_{L}=10$ steps with a time step $\Delta t=0.01$. They are the exact solution of the true system under randomized initial conditions in the form of the Fourier series (4.1). In particular, the initial conditions for both components $u_{1}$ and $u_{2}$ are generated by sampling (4.1) with $a_{0} \sim U\left[-\frac{1}{2}, \frac{1}{2}\right]$, and $a_{n}, b_{n} \sim U\left[-\frac{1}{n}, \frac{1}{n}\right]$ for $1 \leq n \leq N_{c}$ and $N_{c}=10$. A total of 10,000 trajectories are generated for the training data set.

The DNN structure consists of an input layer with 100 neurons, to account for the two components $u_{1}$ and $u_{2}$, each of which has 50 nodes. The disassembly block has dimension $100 \times 1 \times 5$ and the assembly layer has dimension $1 \times 1 \times 5$. The DNN model is trained for 10,000 epochs with a constant learning rate of $10^{-3}$. 

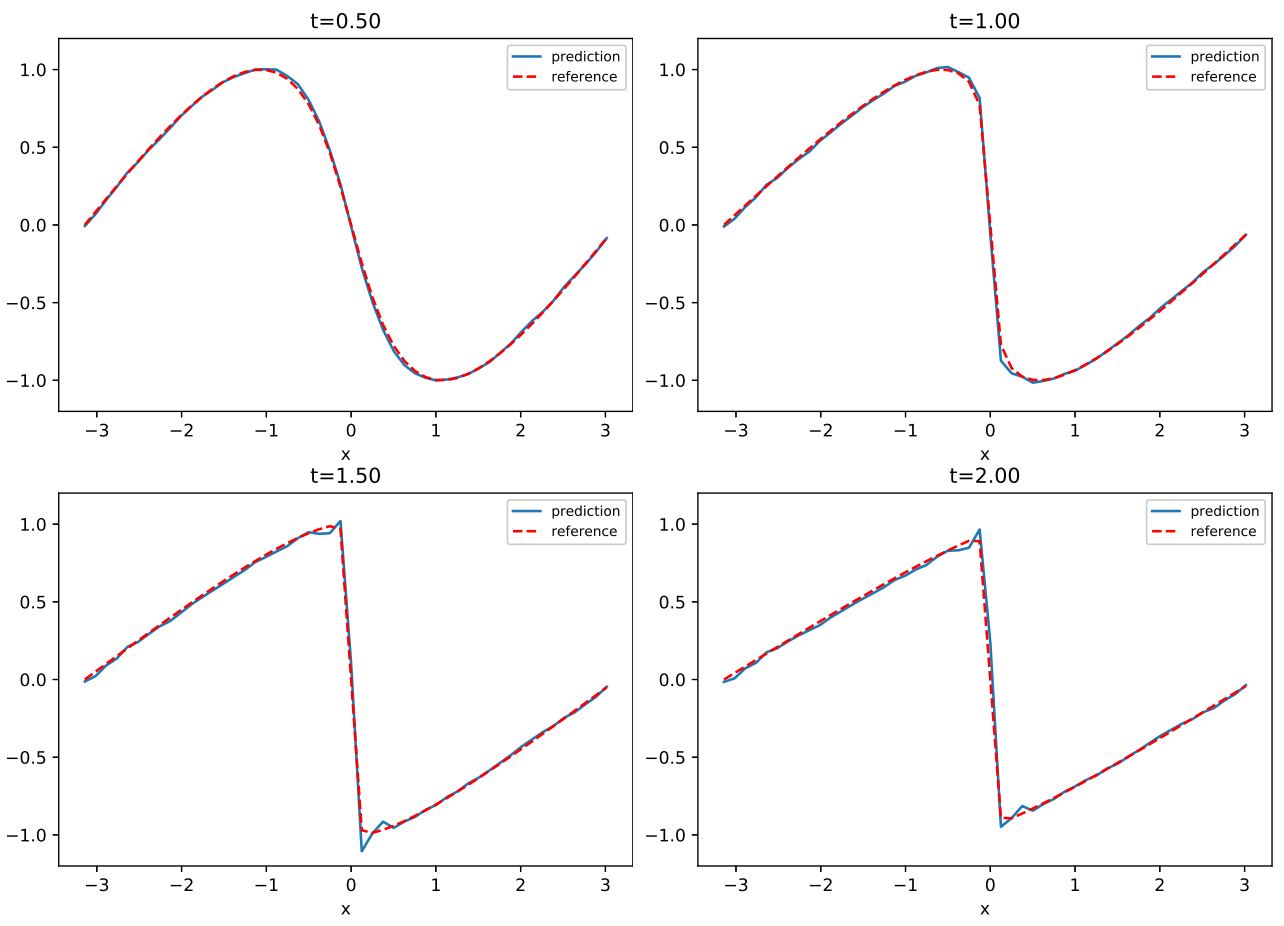

Fig. 4.6: Inviscid Burger's equation. Comparison of DNN model prediction and reference solution. Left to right, up to bottom. Note in this case shock develops at $t=2$.

A set of prediction results generated by the trained DNN model are shown in Fig. 4.7. These are based on a new initial condition

$$
\mathbf{u}(x, 0)=\left[\begin{array}{l}
\exp (\sin (x)) \\
\exp (\cos (x))
\end{array}\right] .
$$

Compared to the true solution, we observe that the DNN model produces accurate prediction for time up to $t=10$.

4.6. Two Dimensional Example. We now consider a two-dimensional advectiondiffusion equation

$$
\frac{\partial u}{\partial t}+\nabla \cdot(\boldsymbol{\alpha} u)=\nabla \cdot(\kappa \nabla u)
$$

on an unstructured grid over domain $(x, y) \in[-1,1] \times[-1,1]$ with zero Dirichlet boundary condition. The transport velocity field is set as $\boldsymbol{\alpha}(x, y)=(y,-x)^{T}$, and the viscosity is set as $\kappa=5 \times 10^{-3}$.

The unstructured grids are shown in Figure 4.8. They consist of 200 points in the interior $(-1,1) \times(-1,1), 8$ points along each edge (resulting 32 points over the edges), and the 4 corner points. The interior points are generated using 2D Sobol sequence in $[0, \pi] \times[0, \pi]$, followed by cosine transformation. The edge points are generated by uniform random distribution in $(0, \pi)$, followed by cosine transformation. 

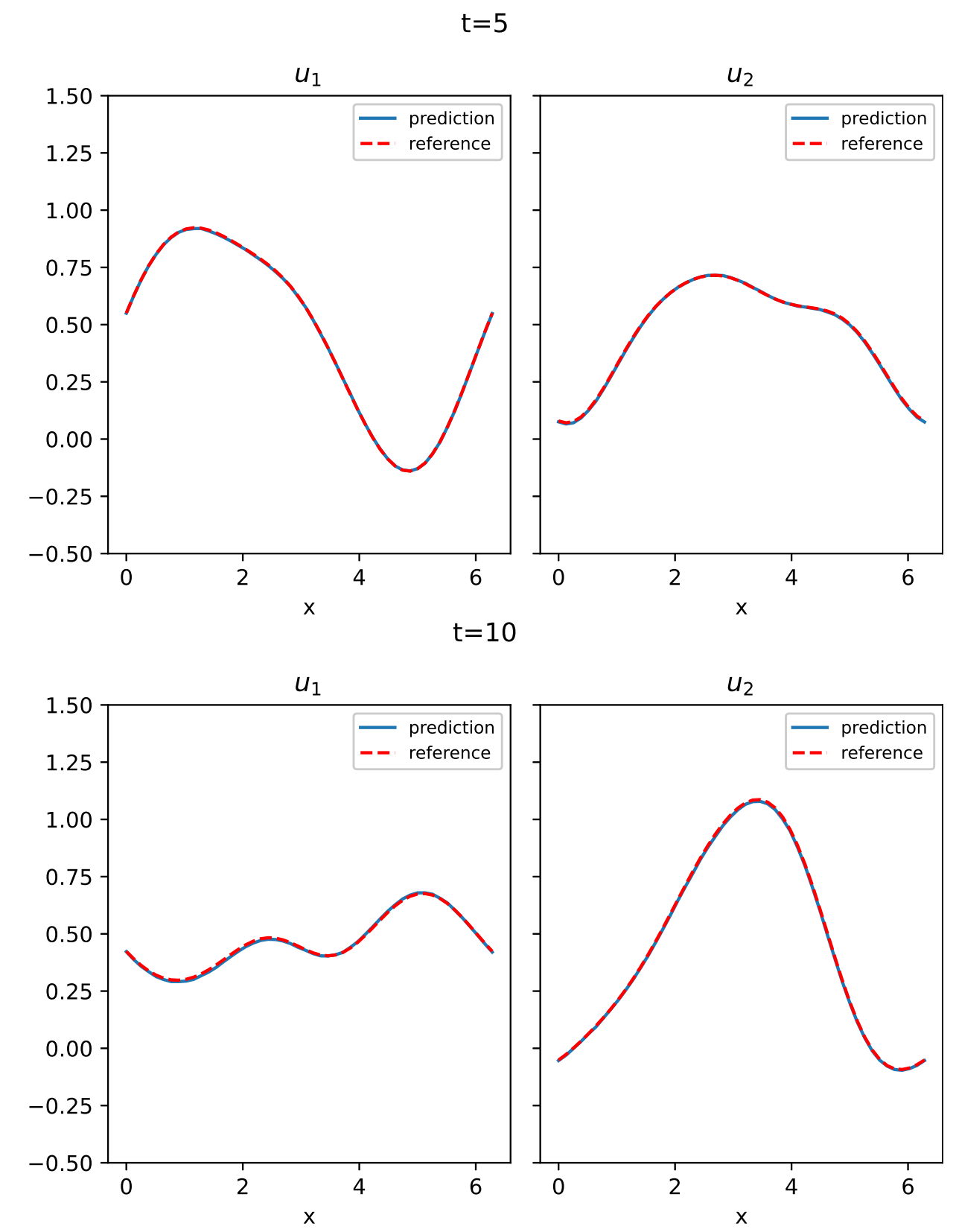

Fig. 4.7: Systems of wave equations. Comparison between DNN model prediction and reference solution.

We generate 100, 000 trajectory data for training using initial condition

$$
u_{0}(x, y)=\sum_{k=1}^{N_{c}} \sum_{l=1}^{N_{c}} c_{k, l} \sin \left(\frac{k \pi}{2}(x+1)\right) \sin \left(\frac{l \pi}{2}(y+1)\right)
$$


where $N_{c}=7$, and the coefficient $c_{k, l}^{(i)} \sim \frac{1}{k+l} U[-1,1]$. The training trajectory data are of length $n_{L}=10$ with time step $\Delta t=2 \times 10^{-3}$, resulting training data of length $t=0.02$.

The DNN has disassembly block with dimension $236 \times 1 \times 3$ and assembly layer of dimension $1 \times 1 \times 3$. The tanh activation function is used throughout, along with a constant learning rate of $1 \times 10^{-3}$. The DNN model is trained for 6,000 epochs with batch size 100 .

For validation, we present the DNN prediction with a new initial condition

$$
u_{0}(x, y)=\frac{C}{\sqrt{4 \pi^{2} \sigma_{x}^{2} \sigma_{y}^{2}}} \exp \left[-\frac{1}{2} \frac{\left(x-\mu_{x}\right)^{2}}{\sigma_{x}^{2}}-\frac{1}{2} \frac{\left(y-\mu_{y}\right)^{2}}{\sigma_{y}^{2}}\right],
$$

where $C=0.2, \mu_{x}=\mu_{y}=0.2$, and $\sigma_{x}=\sigma_{y}=0.18$. In Figure 4.9, we plot the solutions at different time.. We observe good agreement between the DNN model prediction and the reference solution for up to $t=4$.

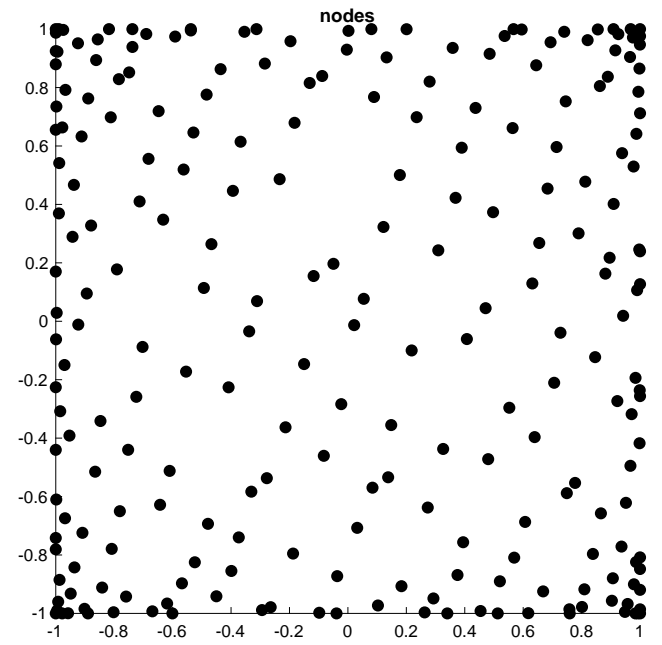

Fig. 4.8: 2D advection-diffusion. Unstructured grids.

5. Conclusion. We present a general framework for DNN learning of unknown PDEs using nodal values of data. A new DNN structure is developed, and its existence is established mathematically. An extensive set of examples are presented and demonstrate that the proposed DNN approach is able to model a variety of model PDE problems from data, particularly unstructured data. The current work introduces an alternative direction for data driven modeling for PDE related problems. More in-depth work on the proposed DNNs will be pursued in a future work.

\section{REFERENCES}

[1] S. L. Brunton, J. L. Proctor, and J. N. Kutz, Discovering governing equations from data by sparse identification of nonlinear dynamical systems, Proc. Natl. Acad. Sci. U.S.A., 113 (2016), pp. 3932-3937.

[2] X. Fu, L.-B. Chang, And D. XiU, Learning reduced systems via deep neural networks with memory, J. Machine Learning Model. Comput., 1 (2020), pp. 97-118. 

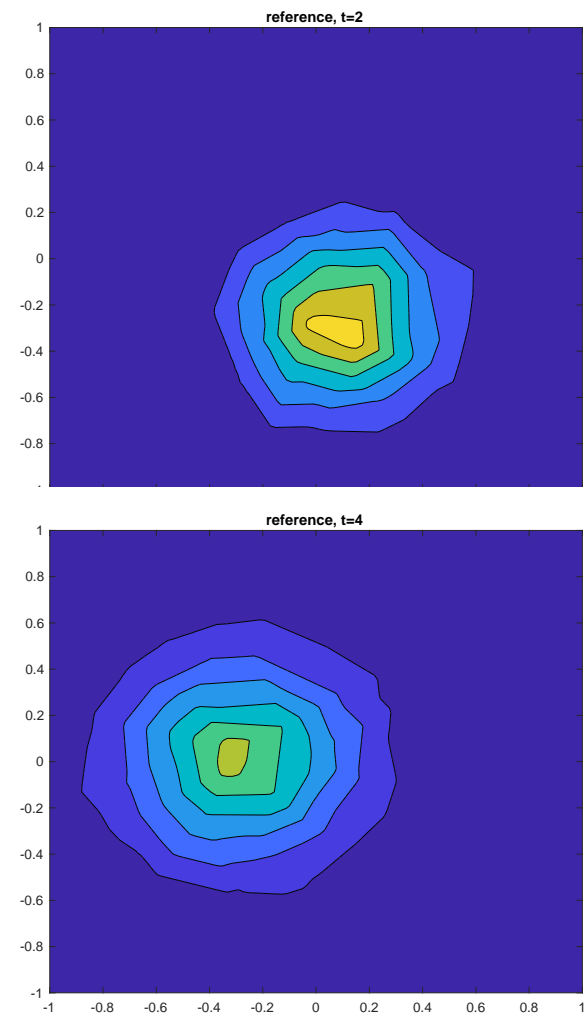
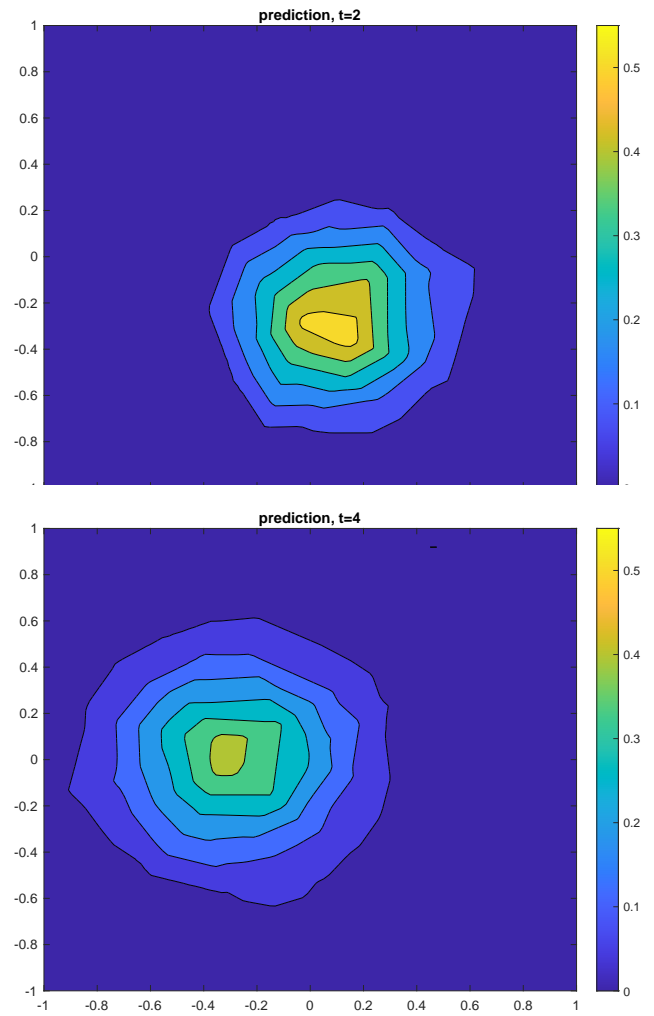

Fig. 4.9: 2D advection-diffusion over unstructured grids: Comparison of DNN model prediction (left column) and reference solution (right column).

[3] K. He, X. Zhang, S. Ren, and J. Sun, Deep residual learning for image recognition, in Proceedings of the IEEE conference on computer vision and pattern recognition, 2016, pp. $770-778$.

[4] S. H. Kang, W. LiaO, AND Y. Liu, IDENT: Identifying differential equations with numerical time evolution, arXiv preprint arXiv:1904.03538, (2019).

[5] Z. Long, Y. Lu, AND B. Dong, PDE-Net 2.0: Learning PDEs from data with a numericsymbolic hybrid deep network, arXiv preprint arXiv:1812.04426, (2018).

[6] Z. Long, Y. Lu, X. MA, AND B. Dong, PDE-net: Learning PDEs from data, in Proceedings of the 35th International Conference on Machine Learning, J. Dy and A. Krause, eds., vol. 80 of Proceedings of Machine Learning Research, Stockholmsmässan, Stockholm Sweden, 1015 Jul 2018, PMLR, pp. 3208-3216.

[7] T. QIn, Z. Chen, J. Jakeman, And D. XIU, Deep learning of parameterized equations with applications to uncertainty quantification, Int. J. Uncertainty Quantification, (2020), p. 10.1615/Int.J.UncertaintyQuantification.2020034123.

[8] T. Qin, Z. Chen, J. Jakeman, And D. Xiu, Data-driven learning of non-autonomous systems, SIAM J. Sci. Comput., (2021), p. in press.

[9] T. QIN, K. WU, AND D. XIU, Data driven governing equations approximation using deep neural networks, J. Comput. Phys., 395 (2019), pp. 620 - 635.

[10] M. RAIssi, Deep hidden physics models: Deep learning of nonlinear partial differential equations, Journal of Machine Learning Research, 19 (2018), pp. 1-24.

[11] M. Raissi, P. Perdikaris, and G. E. Karniadakis, Physics informed deep learning (part i): Data-driven solutions of nonlinear partial differential equations, arXiv preprint arXiv:1711.10561, (2017).

[12] M. Raissi, P. Perdikaris, and G. E. Karniadakis, Physics informed deep learning (part ii): Data-driven discovery of nonlinear partial differential equations, arXiv preprint 
arXiv:1711.10566, (2017).

[13] M. Raissi, P. Perdikaris, And G. E. Karniadakis, Multistep neural networks for data-driven discovery of nonlinear dynamical systems, arXiv preprint arXiv:1801.01236, (2018).

[14] S. H. Rudy, S. L. Brunton, J. L. Proctor, And J. N. Kutz, Data-driven discovery of partial differential equations, Science Advances, 3 (2017), p. e1602614.

[15] S. H. Rudy, J. N. Kutz, And S. L. Brunton, Deep learning of dynamics and signal-noise decomposition with time-stepping constraints, J. Comput. Phys., 396 (2019), pp. 483-506.

[16] F. Scarselli and A. C. Tsoi, Universal approximation using feedforward neural networks: A survey of some existing methods, and some new results, Neural networks, 11 (1998), pp. $15-37$.

[17] H. SCHAEFFER, Learning partial differential equations via data discovery and sparse optimization, Proceedings of the Royal Society of London A: Mathematical, Physical and Engineering Sciences, 473 (2017).

[18] H. Schaeffer and S. G. McCalla, Sparse model selection via integral terms, Phys. Rev. E, 96 (2017), p. 023302.

[19] H. Schaeffer, G. Tran, AND R. WARD, Extracting sparse high-dimensional dynamics from limited data, SIAM Journal on Applied Mathematics, 78 (2018), pp. 3279-3295.

[20] Y. Sun, L. Zhang, And H. Schaeffer, NeuPDE: Neural network based ordinary and partial differential equations for modeling time-dependent data, arXiv preprint arXiv:1908.03190, (2019).

[21] G. Tran And R. WARD, Exact recovery of chaotic systems from highly corrupted data, Multiscale Model. Simul., 15 (2017), pp. 1108-1129.

[22] K. WU, T. QIN, AND D. XIU, Structure-preserving method for reconstructing unknown hamiltonian systems from trajectory data, arXiv preprint arXiv:1905.10396, (2019).

[23] K. WU AND D. XIU, Numerical aspects for approximating governing equations using data, J. Comput. Phys., 384 (2019), pp. 200-221.

[24] K. Wu And D. XIU, Data-driven deep learning of partial differential equations in modal space, J. Comput. Phys., 408 (2020), p. 109307. 